\title{
Hybrid Evolutionary Optimization Algorithm MPSO-SA
}

\author{
Norelislam El Hami ${ }^{1,2, a}$, Rachid Ellaia ${ }^{1}$, Mhamed Itmi $^{2}$ \\ ${ }^{1}$ Laboratory of Study and Research in Applied Mathematics (LERMA), Mohammed V University - Engineering Mohammedia School \\ Rabat, BP. 765 Ibn Sina avenue, Agdal, Morocco \\ ${ }^{2}$ Laboratory (LITIS) of Rouen, National Institute for Applied Sciences - Rouen BP. 08, University Avenue 76801, St Etienne du \\ Rouvray Cedex, France
}

Received 10 December 2009, Accepted 15 February 2010

\begin{abstract}
This paper proposes a new method for a modified particle swarm optimization algorithm (MPSO) combined with a simulated annealing algorithm (SA). MPSO is known as an efficient approach with a high performance of solving optimization problems in many research fields. It is a population intelligence algorithm inspired by social behavior simulations of bird flocking. Considerable research work on classical method PSO (Particle Swarm Optimization) has been done to improve the performance of this method. Therefore, the proposed hybrid optimization algorithms MPSOSA use the combination of MPSO and simulated annealing SA. This method has the advantage to provide best results comparing with all heuristics methods PSO and SA. In this matter, a benchmark of eighteen well-known functions is given. These functions present different situations of finding the global minimum with gradual difficulties. Numerical results presented, in this paper, show the robustness of the MPSO-SA algorithm. Numerical comparisons with these three algorithms: Simulated Annealing, Modified Particle swarm optimization and MPSO-SA prove that the hybrid algorithm offers best results.
\end{abstract}

Key words: Global optimization; PSO; SA; evolutionary algorithm; Hybrid Methods

\section{Introduction}

The Particle Swarm Optimization (PSO), developed by Kennedy and Eberharts in 1995 [22], is an approximation algorithm method proposed for the optimization problem of finding the global minimum. Since then, it has been improved by many searchers. The principal of this algorithm is based on the movement of birds searching for a food in a flock; this animal behavior is simulated to the optimization research.

This method generates a group of particles, each one search for the minimum of the fitness by their own knowledge and movement, and is influenced by the search of his neighbor. If a particle finds a good site, all the others can become aware of it more or less directly, in order to take advantage of it.

Bochenek Fory have improved particle swarm optimizer in 2006 [19] by adding a new term in the velocity equation. It represents the distance between the particle position and the position of the particle neighbors' leader, the best particle among its neighbors. The complementary information provided influences swarm member behavior and, in many cases, their algorithm can improve swarm performance.

A simulated annealing algorithm SA is based on the idea in physics of annealing a solid to the state with a minimum energy. In this algorithm an initial solution is generated which is repeatedly improved by making a few alterations. This process had been already done in a con-

\footnotetext{
${ }^{a}$ Corresponding author: norelislam@hotmail.com
}

stant temperature using the probability test for accepting a candidate solution which depends on the individual estimated objective function value. The algorithm is shown to converge almost surely to an optimal solution.

The modified particle swarm optimization algorithm (MPSO)-simulated annealing algorithm SA (MPSO-SA) used modified PSO as an evolutionary searching mechanism to effectively perform exploration for promising solutions within the entire region, and used SA to perform exploitation for solution improvement. Several combinations have been tested to avoid selecting an appropriate model.

Majid Bahrepour has presented, in 2009 [8], a new method called SUPER-SAPSO algorithm which fuses SA with standard PSO. The idea of this method is that the new position during the movement of the particle in the swarm is multiplied by the temperature factor and the SUPER-SAPSO assigns the temperatures locally to each particle of the swarm. The temperature is a function of error, they assign a hot temperature to particles with poorer fitness.

Behnamian and Ghomi have published, in 2010 [1], a hybrid method where SA-based local search is applied to the best solution given during the PSO search process. At high temperature, the algorithm performs exploration with certain jumping probability while at low temperature, the algorithm stresses the exploitation from the best solution. 


\section{Nomenclature and Abbreviations .}

SH3 Shubert function with 3 dimension

SH4 Shubert function with 4 dimension

BO1 Bohachevsky1 function

BO2 Bohachevsky2 function

BR Branin function

CA Camel function

CM Cosine Mixture function

DE Dejoung function

GP Goldstein Price function

GR Griewank Price function

HAN Hansen function

HA3 Hartman function, 3 dimension

HA6 Hartman function, 6 dimension

RA Rastrigin function

RO Rosenbrock function

SK5 Shekel function

SK7 Shekel function

SK10Shekel function

Dim Function dimension

SR Success Rate

SD Standard Deviation

EvalFNumber of Evaluated Function

Ftest Test Function

PSO Particle Swarm Optimization

SA Simulated Annealing

\section{SA Algorithm}

SA is a classical optimization technique that has been successfully used for solving a wide range of optimization problems. In the SA algorithm, like most of its family (Meta heuristics that are based on gradual local improvement), we started with a randomly chosen none optimal configuration and moved iteratively to another solution in the neighborhood to improve the configuration. A brief description of this algorithm is given in this section in order to show the combination with the PSO in later sections. In the first stage, the algorithm started from an initial solution $\mathrm{S}$ which is generated randomly, thereafter we generated a new solution $S$ ' in the neighborhood of the precedent one. For minimization problems, the new solution is accepted with certain probability $\mathrm{P}$.

The probability $P<\exp \left(\frac{\Delta E}{T}\right)$

where $\Delta \mathrm{E}$ is the variation of the energy, which can also be the variation of the fitness in optimization method.

$\Delta \mathrm{E}=\mathrm{F}\left(\mathrm{S}^{\prime}\right)-\mathrm{F}(\mathrm{S})$. $\mathrm{T}$ is the temperature and it is considered in this study as the control parameter. This probability principle is used to select the uphill moves that may help the optimization procedure escape from local minima. In this algorithm we started from high temperature value to a lower one gradually in order to find precisely the global optimum.
Pseudocode of Simulated Annealing algorithm .

.$S \leftarrow$ Sinit Generate the initial population randomly.

.Sbest $\leftarrow S$

.Fbest $\leftarrow F(S)$

.Initialisation of $\mathrm{T}$ (temperature)

.Start Iterations NbIter $\leftarrow 0$

.While $T>$ Tminimum

NbIter $\leftarrow$ NbIter +1

- Generate $S^{\prime}$ in the neighbour of $S$

. $\Delta \leftarrow F\left(S^{\prime}\right)-F(S)$

. if $\Delta<0$

$S \leftarrow S^{\prime}$

if $F\left(S^{\prime}\right)<F($ Sbest $)$

Fbest $\leftarrow F\left(S^{\prime}\right)$

Sbest $\leftarrow S^{\prime}$

$$
\text { end }
$$

else

$$
\text { Random } p \text { in [0 1] }
$$

if $p \leq \exp (-\Delta / T)$

$S \leftarrow S^{\prime}$

end

- end

.end

\section{MPSO Algorithm}

In Particle Swarm Algorithm, each particle i is treated as a point in a space with dimension $\mathrm{D}$, a position $X_{i}$, a velocity $V_{i}$ and personal best position $X_{\text {best }}$. The personal best position associated with a particle $\mathrm{i}$ is the best position that the particle has visited. The best position of all particles in the swarm is represented by the vector $X_{\text {gbest }}$.

$X_{i}=\left(x_{i 1}, x_{i 2}, \ldots \ldots, x_{i d}\right)$ The position of the particle.

$V_{i}=\left(v_{i 1}, v_{i 2}, \ldots \ldots, v_{i d}\right)$ The velocity of the particle.

$X_{\text {besti }}=\left(p_{i 1}, p_{i 2}, \ldots \ldots, p_{i d}\right)$ The best personnel position.

$X_{\text {gbest }}=\left(p_{g 1}, p_{g 2}, \ldots \ldots, p_{g d}\right)$ The best global position.

$$
\begin{array}{r}
V_{i d}(t+1)=\chi\left(V_{i d}(t)+\rho_{1}\left[X_{\text {besti }}(t)-X_{i}(t)\right]\right. \\
\left.+\rho_{2}\left[X_{\text {gbest }}(t)-X_{i}(t)\right]\right) \\
X_{i d}(t+1)=X_{i d}(t)+V_{i d}(t+1)
\end{array}
$$

$\rho_{1}=c_{1} r_{1}$ and $\rho_{2}=c_{2} r_{2}$

$c_{1}$ and $c_{2}$ : positive acceleration components called social parameter .

$\chi$ : constriction coefficient.

$r_{1}$ and $r_{2}$ : Independent random number in the rang $[0,1]$

We modified the velocity function by using a new term $X_{N \text { best }}$ in the equation 1 , which was introduced by Bochenek and Fory [19] defined as:

$X_{\text {Nbest }}=\left(p_{n 1}, p_{n 2}, \ldots \ldots, p_{n d}\right)$ The best position of the neighborhood. 
The equation 1 becomes :

$$
\begin{gathered}
V_{i d}(t+1)=\chi\left(V_{i d}(t)+\rho_{1}\left[X_{\text {besti }}(t)-X_{i}(t)\right]+\right. \\
\left.\rho_{2}\left[X_{\text {gbest }}(t)-X_{i}(t)\right]+\rho_{3}\left[X_{\text {Nbest }}(t)-X_{i}(t)\right]\right)
\end{gathered}
$$

$\rho_{3}=c_{3} r_{3}$

$c_{3}$ : positive acceleration components called social parameter.

$r_{3}:$ Independent random number in the rang $[0,1]$

The initialization of the swarm and velocities is usually performed randomly in the search space, following a uniform distribution. The best positions are initially set equal to the initial swarm. After the first time increment, they will be moved by the velocity $V_{i}$ in Equation (3). Then the algorithm searches for optima by updating generations. The acceleration constants $c_{1}, c_{2}$ and $c_{3}$ in Equation (3) represent the weighting of the stochastic acceleration terms that pull each particle towards $X_{\text {besti }}$, $X_{N b e s t}$ and $X_{\text {gbest }}$ positions. $c_{1}$ represents the confidence that the particle has in itself, $c_{2}$ represents the confidence that the particle has in the swarm, and $c_{3}$ represents the confidence that the particle has in his neighbor. In most cases, the acceleration parameters $c_{1}, c_{2}$ and $c_{3}$ are affected to 1 , however, if we want to eliminate the particle's own experience we take $c_{1}=0 ; c_{2}=1$ and $c_{3}=1$ or eliminate the influence of the best of the swarm we take $c_{1}=1 ; c_{2}=0$ and $c_{3}=1$ or we eliminate the influence of the best of the neighbor we take $c_{1}=1 ; c_{2}=1$ and $c_{3}=0$. Depending on the problems to resolve we can make the appropriate choices for these parameters to modify the velocity and to promote convergence.

The search procedure of a population-based algorithm such as PSO consists on the concept of neighborhood, the information regarding the best position of each neighborhood is gradually communicated to the rest of the particles through their neighbors in the ring topology, we have a neighborhoods that consist of particles belong to different partitions. In this case, particles with different behaviors can interact by sharing information through their neighborhoods. All particles in a neighbor share the same value of $X_{N b e s t}$ and each neighbor has a different value of $X_{\text {Nbest }}$. It is important to respect the number of particles that comprise the neighborhoods, therefore, in our experiments the swarm was divided into 7 partitions. In general there is no formal procedure to determine the optimal number or the size of the neighbor but case by case depending on the problems to resolve.

Pseudocode of Modified Particle Swarm Optimization.

Initialization (Randomly)

. $X_{i} \leftarrow$ Generate the initial particles of the swarm .$V_{i} \leftarrow$ Generate the initial velocity of the particles .$X_{\text {best } i} \leftarrow X_{i}$ Set the best positions of the particle .$X_{\text {gbest }} \leftarrow X_{i}$ Set the best positions of the swarm .$X_{N b e s t} \leftarrow X_{i}$ Set the best positions of the neighbor

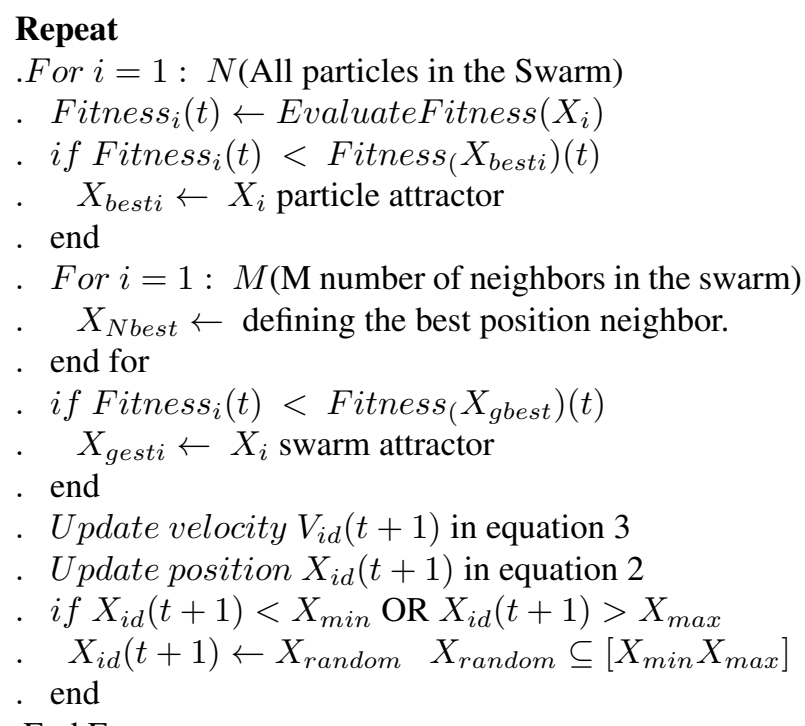

.End For

Until Stop criterion

\section{Proposed MPSO-SA Algorithm}

In this study, a new hybrid evolutionary algorithm is proposed which incorporate the SA algorithm into a MPSO. Firstly, we have modified PSO by using three terms in the velocity equation and then we combined it with the SA algorithm to increase the diversity of the population and to improve the convergence. In the proposed algorithm called MPSO-SA, SA is used as a local search around the two best positions, the first one is the best particle in the neighborhood $X_{N b e s t}$ and the second one is the best position in the swarm $X_{\text {gbest }}$.

The simulation of the proposed hybrid algorithm begins with an initial population and initial temperature. The particles then randomly search according to evolutionary equations of MPSO algorithm to generate a new population, which is compared and improved by SA algorithm. Then the results obtained become the individuals of the next generation. The simulation is repeated until the terminal criterion is met, which is reached when there is no improvement of the solution.

The simulated annealing is adapted to be used in the hybridization: the number of iteration in SA is reduced to increase the performance. Also the temperature parameter $\mathrm{T}$ is decreased within the MPSO algorithm, in each iteration a new value of the temperature and the best positions are given to SA, which start his search around them. The mechanism starts with a high value of the temperature, so we accept a given value of the best positions, after that and during the process the temperature decreases and the search are directed towards those positions that have shown a relative advantage over others, and in the same time to guide the swarm with the probability to further increase and cover the search space.

The general procedure for the MPSO-SA algorithm can be summarized as follows: 
Pseudocode of MPSO-SA .

\section{Initialization (Rondomly)}

.$X_{i} \leftarrow$ Generate the initial particles of the swarm .$V_{i} \leftarrow$ Generate the initial velocity of the particles $X_{\text {besti }} \leftarrow X_{i}$ Set the best position of the particle .$X_{\text {gbest }} \leftarrow X_{i}$ Set the best position of the swarm .$X_{\text {Nbest }} \leftarrow X_{i}$ Set the best position of the neighbor Initialisation of $\mathrm{T}$ (temperature)

\section{Repeat}

.For $i=1: N$

. Fitness $s_{i}(t) \leftarrow$ EvaluateFitness $\left(X_{i}\right)$

. if Fitness $s_{i}(t)<$ Fitness $\left._{(} X_{\text {besti }}\right)(t)$

$X_{\text {besti }} \leftarrow X_{i}$ particle attractor

end

- if Fitness ${ }_{i}(t)<$ Fitness $\left._{(} X_{\text {gbest }}\right)(t)$

$X_{\text {gbest }} \leftarrow X_{i}$ swarm attractor

end

Start SA

For $i=1: M(\mathrm{M}$ number of neighbors in the swarm)

$X_{\text {Nbest }} \rightarrow$ SA algorithm

$X_{N b e s t} \leftarrow$ modify the best positions of the neighbor end for

End SA

- Update velocity $V_{i d}(t+1)$ in equation 3

Update position $X_{i d}(t+1)$ in equation 2

. if $X_{i d}(t+1)<X_{\min }$ OR $X_{i d}(t+1)>X_{\max }$ $X_{i d}(t+1) \leftarrow X_{\text {random }} \quad X_{\text {random }} \subseteq\left[X_{\text {min }} X_{\text {max }}\right]$ end

. Update the temperature value

.end

Until Stop criterion

\section{Main results of Benchmark.}

To illustrate the performance of the proposed MPSO-SA, we compare it with MPSO and standard SA, some investigation on benchmark functions are reported in the following tables. These functions are standard benchmark with different sizes and are all minimization problems. In order to give right indications of relative performance for each method, 100 runs were performed in this experimental setting. The results are summarized in the following with the average values.
Table 1: Simulated annealing results

\begin{tabular}{cccccc}
\hline & & \multicolumn{3}{c}{ SA } \\
\cline { 3 - 6 } FTest & Dim & SR & SD & Time(s) & EvalF \\
\hline SH3 & 3 & 80 & 10.7 & 40 & 1342250.6 \\
\hline SH4 & 4 & 20 & $6.76 \mathrm{e}+003$ & 69 & 2074982.6 \\
\hline GR & 50 & 10 & 17.0684 & $9.6 \mathrm{e}+002$ & 2814728.3 \\
\hline RO & 10 & 23 & 44.9 & 78 & 2796580.5 \\
\hline HAN & 2 & 100 & 0.0308 & 50 & 1945584.2 \\
\hline SK5 & 4 & 60 & 1.7 & $2.1 \mathrm{e}+002$ & 2205679.4 \\
\hline SK7 & 4 & 70 & 0.436 & $2.8 \mathrm{e}+002$ & 2195135 \\
\hline SK10 & 4 & 100 & 0.00227 & $3.6 \mathrm{e}+002$ & 2104962.2 \\
\hline HA3 & 3 & 100 & $9.02 \mathrm{e}-005$ & 85 & 2440969 \\
\hline HA6 & 6 & 20 & 0.00684 & $5.6 \mathrm{e}+002$ & 13014268.2 \\
\hline DE & 3 & 100 & $6.59 \mathrm{e}-006$ & 47 & 2463189 \\
\hline CA & 2 & 100 & $7.45 \mathrm{e}-007$ & 52 & 2463189 \\
\hline GP & 2 & 100 & $9.49 \mathrm{e}-006$ & 51 & 2272379.8 \\
\hline CM & 4 & 50 & 0.000296 & 55 & 2438221.8 \\
\hline BR & 2 & 100 & $4.53 \mathrm{e}-007$ & 49 & 2463189 \\
\hline BO1 & 2 & 100 & $6.65 \mathrm{e}-007$ & 49 & 2463189 \\
\hline BO2 & 2 & 100 & $4.06 \mathrm{e}-006$ & 49 & 2463189 \\
\hline RA & 2 & 30 & 0.00447 & 48 & 2305790.6 \\
\hline
\end{tabular}

Table 2: Modified Particle Swarm Optimisation results

\begin{tabular}{cccccc}
\hline & & \multicolumn{4}{c}{ MPSO } \\
\cline { 3 - 6 } FTest & Dim & SR & SD & Time(s) & EvalF \\
\hline SH3 & 3 & 100 & $3.58 \mathrm{e}-012$ & 3.6 & 229284.02 \\
\hline SH4 & 4 & 100 & $1.05 \mathrm{e}-011$ & 4.6 & 269959.4 \\
\hline GR & 50 & 40 & 0.446 & 20 & 582084.6 \\
\hline RO & 10 & 62 & 1.64 & 7.1 & 476140 \\
\hline HAN & 2 & 100 & $1.27 \mathrm{e}-014$ & 3.1 & 241828.2 \\
\hline SK5 & 4 & 50 & 3.13 & 7.7 & 188575.6 \\
\hline SK7 & 4 & 70 & 3.2 & 8.9 & 170397.6 \\
\hline SK10 & 4 & 70 & 3.48 & 10 & 139824.58 \\
\hline HA3 & 3 & 100 & $6.49 \mathrm{e}-015$ & 4.6 & 248340.8 \\
\hline HA6 & 6 & 51 & 0.0598 & 5.5 & 239534.8 \\
\hline DE & 3 & 100 & 0 & 5.4 & 476140 \\
\hline CA & 2 & 100 & $9.93 \mathrm{e}-017$ & 2 & 156030 \\
\hline GP & 2 & 100 & $5.58 \mathrm{e}-015$ & 2.6 & 203344.4 \\
\hline CM & 4 & 100 & $9.02 \mathrm{e}-015$ & 3.1 & 246113 \\
\hline BR & 2 & 100 & 0 & 1.2 & 99409.8 \\
\hline BO1 & 2 & 100 & 0 & 0.71 & 61088 \\
\hline BO2 & 2 & 100 & 0 & 0.72 & 63182.4 \\
\hline RA & 2 & 100 & 0 & 0.83 & 69802.6 \\
\hline
\end{tabular}


Table 3: Proposed MPSO-SA results

\begin{tabular}{cccccc}
\hline & & \multicolumn{4}{c}{ MPSO-SA } \\
\cline { 3 - 6 } FTest & Dim & SR & SD & Time(s) & EvalF \\
\hline SH3 & 3 & 100 & $3.69 \mathrm{e}-012$ & 3.4 & 137372.08 \\
\hline SH4 & 4 & 100 & $7.4 \mathrm{e}-011$ & 4.3 & 455889.6 \\
\hline GR & 50 & 100 & $1.6 \mathrm{e}-010$ & 18 & 570334.2 \\
\hline RO & 10 & 91 & 1.02 & 4.9 & 243578 \\
\hline HAN & 2 & 100 & $2.6 \mathrm{e}-013$ & 3.1 & 364928 \\
\hline SK5 & 4 & 58 & 5.7 & 6.9 & 256903 \\
\hline SK7 & 4 & 82 & 3.25 & 9.3 & 198789 \\
\hline SK10 & 4 & 83 & 5.76 & 8.6 & 176896 \\
\hline HA3 & 3 & 100 & $6.55 \mathrm{e}-015$ & 2.9 & 80846.1 \\
\hline HA6 & 6 & 61 & 0.132 & 3.4 & 76704.18 \\
\hline DE & 3 & 100 & 0 & 3.4 & 213560 \\
\hline CA & 2 & 100 & $1.57 \mathrm{e}-016$ & 1.9 & 83971.2 \\
\hline GP & 2 & 100 & $1.06 \mathrm{e}-014$ & 2 & 86159.2 \\
\hline CM & 4 & 100 & $1.16 \mathrm{e}-014$ & 0.74 & 32718.4 \\
\hline BR & 2 & 100 & 0 & 1.2 & 57040 \\
\hline BO1 & 2 & 100 & 0 & 0.73 & 34725.9 \\
\hline BO2 & 2 & 100 & 0 & 0.74 & 35043.9 \\
\hline RA & 2 & 100 & 0 & 0.61 & 28264 \\
\hline
\end{tabular}

The results show, in most of the time, that the MPSOSA can achieve the optimal solution with higher probability and the computation time is lower than the other methods SA and MPSO. The SA can guarantee the optimum solution when the problem size is small, but it will take a long time when the problem size is larger. The MPSO can solve the larger problem in an acceptable time, which demonstrates the powerful explore-ability of the MPSO algorithm. We can mention that sometimes it cannot find the optimal solution. The results reveal that the number of function evaluations of MPSO-SA is less than the other algorithms. The comparison strongly suggests that the MPSO-SA is able to efficiently improve the computational performance of complex optimization problems.

The performance of the algorithm is evaluated in comparison with results obtained from other algorithms for a number of benchmark instances. The new algorithm is very effective and efficient. It can find the optima for most test instances and running time is less than almost all other algorithms. Because of the generality of MPSO$\mathrm{SA}$, it can be applied to many optimization problems. These results indicate that the proposed algorithm is an attractive alternative for solving optimization problems.

\section{Conclusion}

In this paper, we presented the hybridization of population-based evolutionary searching ability of our new method MPSO-SA. The results of effective hybrid MPSO-SA was proposed. The results of simulation indicated that the disadvantage of classical PSO is conquered by MPSO method. The ability of global optimality is toned up by MPSO-SA. The balance between the global exploration and the local exploitation was stressed. On one hand, MPSO-SA applied the evolutionary searching mechanism of MPSO which is modified from the classical one and characterized by individual improvement plus population cooperation and competition to effectively perform exploration. On the other hand, MPSO-SA used an adaptive local search by SA method to perform exploitation. The simulation results and comparisons with the three approaches demonstrated the superiority of the proposed MPSO-SA in terms of searching quality, robustness and the low number of function evaluation. Our proposed method is a promising solution and encourages us to apply it to other Optimization problems.

\section{References}

1. J.Behnamian, S.M.T Fatemi Ghomi, Development of a PSO-SA hybrid metaheuristic for a new comprehensive regression model to time-series forecasting, Expert Systems with Applications, 974-984, (2010).

2. V. Savsani, R.V. Rao, D.P. Vakharia, Optimal weight design of a gear train using particle swarm optimization and simulated annealing algorithms, Mechanism and Machine Theory, 531-541, (2010).

3. M.M. Ali, M.N. Gabere, A simulated annealing driven multi-start algorithm for bound constrained global optimization, Journal of Computational and Applied Mathematics, 2661-2674, (2010).

4. T. Niknam, B. Amiri, J. Olamaei, A. Arefi, An efficient hybrid evolutionary optimization algorithm based on PSO and SA for clustering, Journal of Zhejiang University SCIENCE, 512-519, 2009.

5. S. Sitarz, Ant algorithms and simulated annealing for multicriteria dynamic programming, Computers \& Operations Research, 433-441, (2009).

6. Y. Zhaoa, W. Zub, H. Zeng, A modified particle swarm optimization via particle visual modeling analysis, Computers and Mathematics with Applications, 2022-2029, (2009).

7. M.H. Alrefaei, A.H. Diabat, A simulated annealing technique for multi-objective simulation optimization, Applied Mathematics and Computation, 30293035, (2009).

8. M. Bahrepour, E. Mahdipour, R. Cheloi, M. Yaghoobi, SUPER SAPSO: A New SA-Based PSO Algorithm, Applications of Soft Computing, 423430, (2009). 
9. W. Du, B. Li, Multi-strategy ensemble particle swarm optimization for dynamic optimization, Information Sciences, 3096-3109, (2008).

10. L. Lamberti, An efficient simulated annealing algorithm for design optimization of truss structures, Computers and Structures, 1936-1953, (2008).

11. S.W. Lin, T.Y. Tseng, S.Y. Chou, S.C. Chen, A simulated-annealing-based approach for simultaneous parameter optimization and feature selection of back-propagation networks, Expert Systems with Applications, 1491-1499, (2008).

12. L.L. Li, D.H. Zhou, L. Wang, Fault Diagnosis of Nonlinear Systems Based on Hybrid PSOSA Optimization Algorithm, International Journal of Automation and Computing, 183-188, April (2007).

13. B. Liu, L. Wang, Y.H. Jin, An effective hybrid particle swarm optimization for no wait flow shop scheduling, Int J Adv Manuf Technol, 1001-1011, (2007).

14. F. Zhao, Y. Hong, D. Yu, Y. Yang, Q. Zhang, H. Yi, A hybrid algorithm based on particle swarm optimization and simulated annealing to holon task allocation for holonic manufacturing system, Int J Adv Manuf Technol, 1021-1032, (2007).

15. P.S. Shelokar, P. Siarry, V.K. Jayaraman, B.D. Kulkarni, Particle swarm and ant colony algorithms hybridized for improved continuous optimization, Applied Mathematics and Computation, 129-142, (2007).

16. R. Brits, A.P. Engelbrecht, F. van den Bergh, Locating multiple optima using particle swarm optimization, Applied Mathematics and Computation, 18591883, (2007).

17. Y. Jiang, T. Hu, C. Huang, X. Wu, An improved particle swarm optimization algorithm, Applied Mathematics and Computation, 231-239, (2007).

18. Y. Liu, Z. Qin, Z. Shi, J. Lu, Center particle swarm optimization, Neurocomputing, 672-679, (2007).

19. B. Bochenek, P. Foryś, Structural optimization for post buckling behavior using particle swarms, Struct Multidisc Optim, 521-531, (2006).

20. D. Chaojin, Q. Zulian, Particle Swarm Optimization Algorithm Based on the Idea of Simulated Annealing, International Journal of Computer Science and Network Security, 6(10), October (2006).

21. W.J. Xia, Z.M. Wu, A hybrid particle swarm optimization approach for the job shop scheduling problem, Int J Adv Manuf Technol, 360-366, (2006).

22. R.C. Eberhart, J. Kennedy, A new optimizer using particle swarm theory, in: Proceedings Sixth Symposium on Micro Machine and Human Science, IEEE Service Center, Piscataway, NJ, 39-43, (1995). 